\title{
Amphiphilic co-Polypeptides Self-Assembled into Spherical Nanoparticles for Dermal Drug Delivery
}

Vahid Ahmadi, ${ }^{\ddagger}$ Fatemeh Zabihi, ${ }^{\ddagger} \S$ Fiorenza Rancan, $§$ Arthur Alexander Staszak, ${ }^{\ddagger}$ Chuanxiong Nie, ${ }^{\ddagger}$ Mathias Dimde, ${ }^{\ddagger}$ Katharina Achazi, ${ }^{\ddagger}$ Arno Wiehe, ${ }^{\ddagger} \dagger$ Annika Vogt, $\S$ and Rainer Haag ${ }^{*} \ddagger$

† Institut für Chemie und Biochemie, Freie Universität Berlin, Takustrasse 3, 14195 Berlin, Germany. E-mail: haag@chemie.fu-berlin.de

$\S$ Clinical Research Center for Hair and Skin Science, Department of Dermatology and Allergy, CharitéUniversitätsmedizin Berlin, Charitéplatz 1, 10117 Berlin, Germany

† biolitec research GmbH, Otto-Schott-Str. 15, 07745 Jena, Germany<smiles>CC(=O)NCCC(N)C(=O)O</smiles>

(1 eq)<smiles>O=C(OC(Cl)(Cl)Cl)OC(Cl)(Cl)Cl</smiles>

(0.6-1 eq)

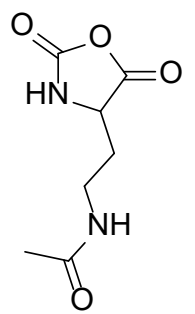

Yield $<10 \%$

Scheme $\mathbf{S}$ 1. Synthesis of NCA-NADA using triphosgene.

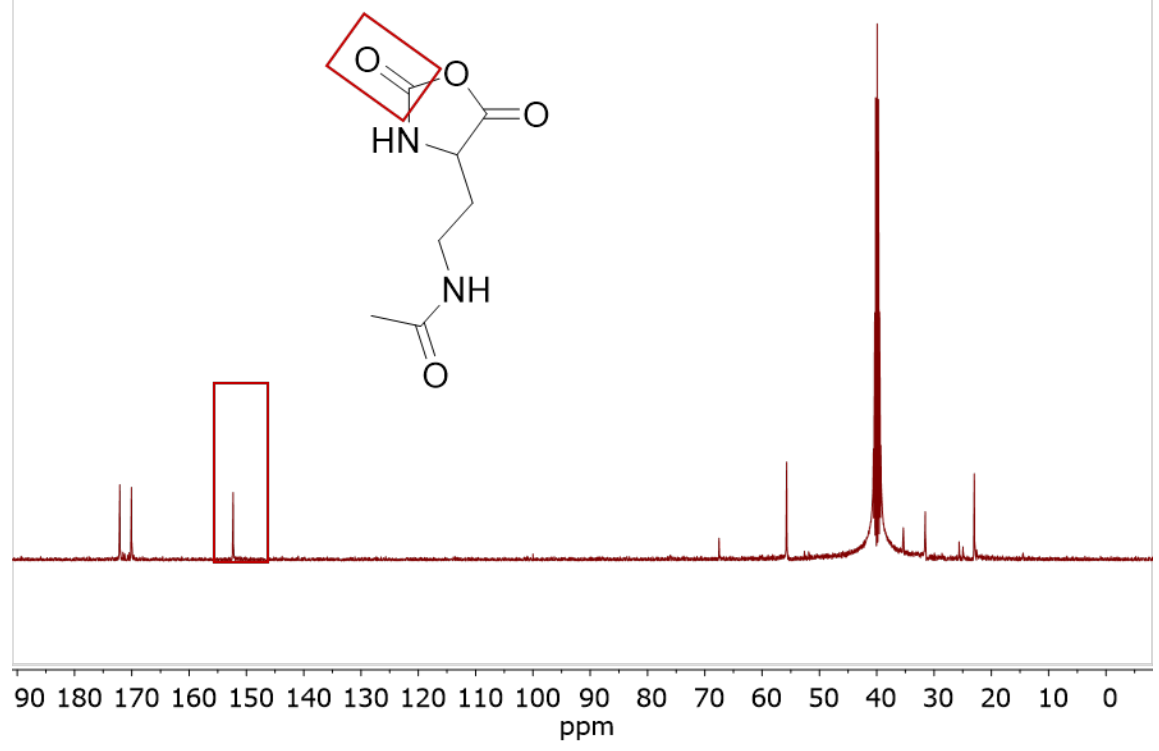

Figure S 1. ${ }^{13} \mathrm{C}$ NMR of NCA-NADA in DMSO- $\mathrm{d}_{6}$ 

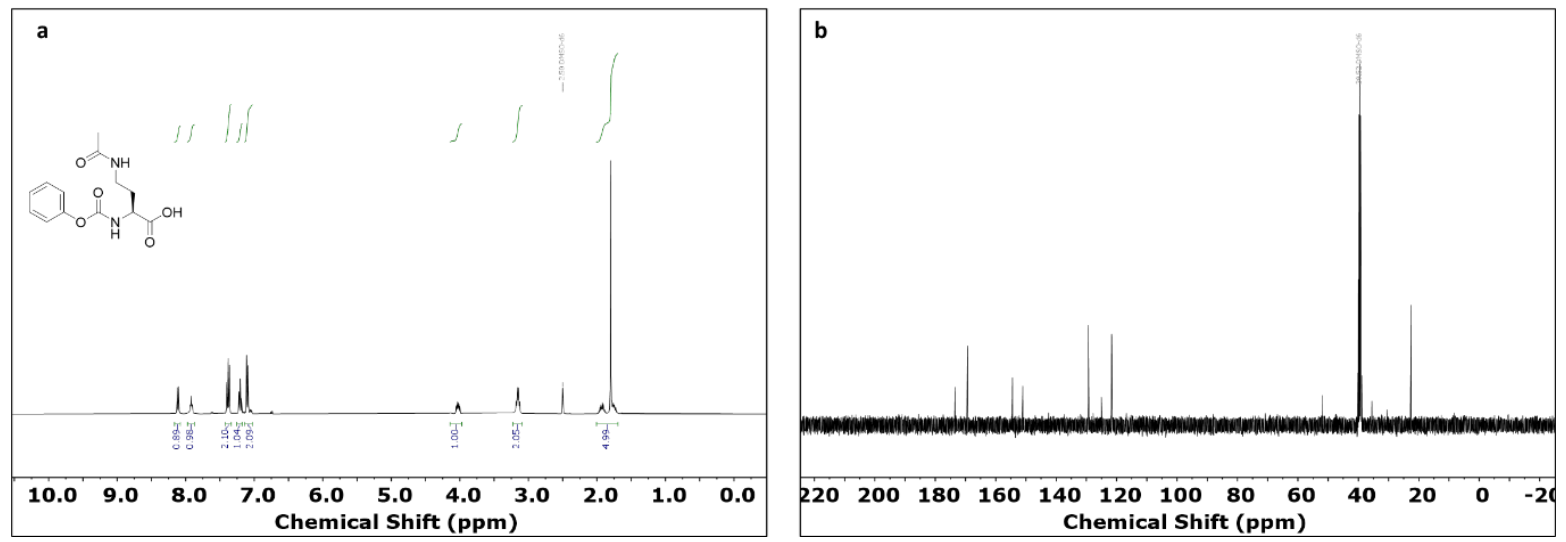

Figure S 2. (a) ${ }^{1} \mathrm{H}$ NMR (b) and ${ }^{13} \mathrm{C}$ NMR spectra of Ph-NADA in dmso- $\mathrm{d}_{6}$.

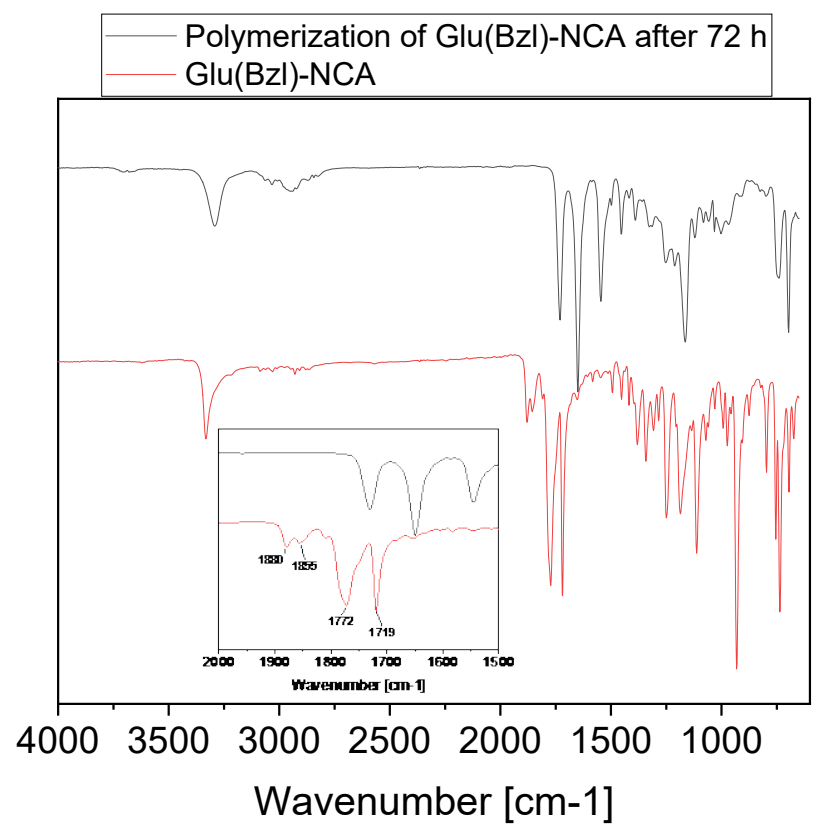

Figure S 3. The removal of the signal intensity of the NCA-associated carbonyl vibrational band at $1772 \mathrm{~cm}^{-1}$ after 3 days of NCA polymerization observed via IR. 


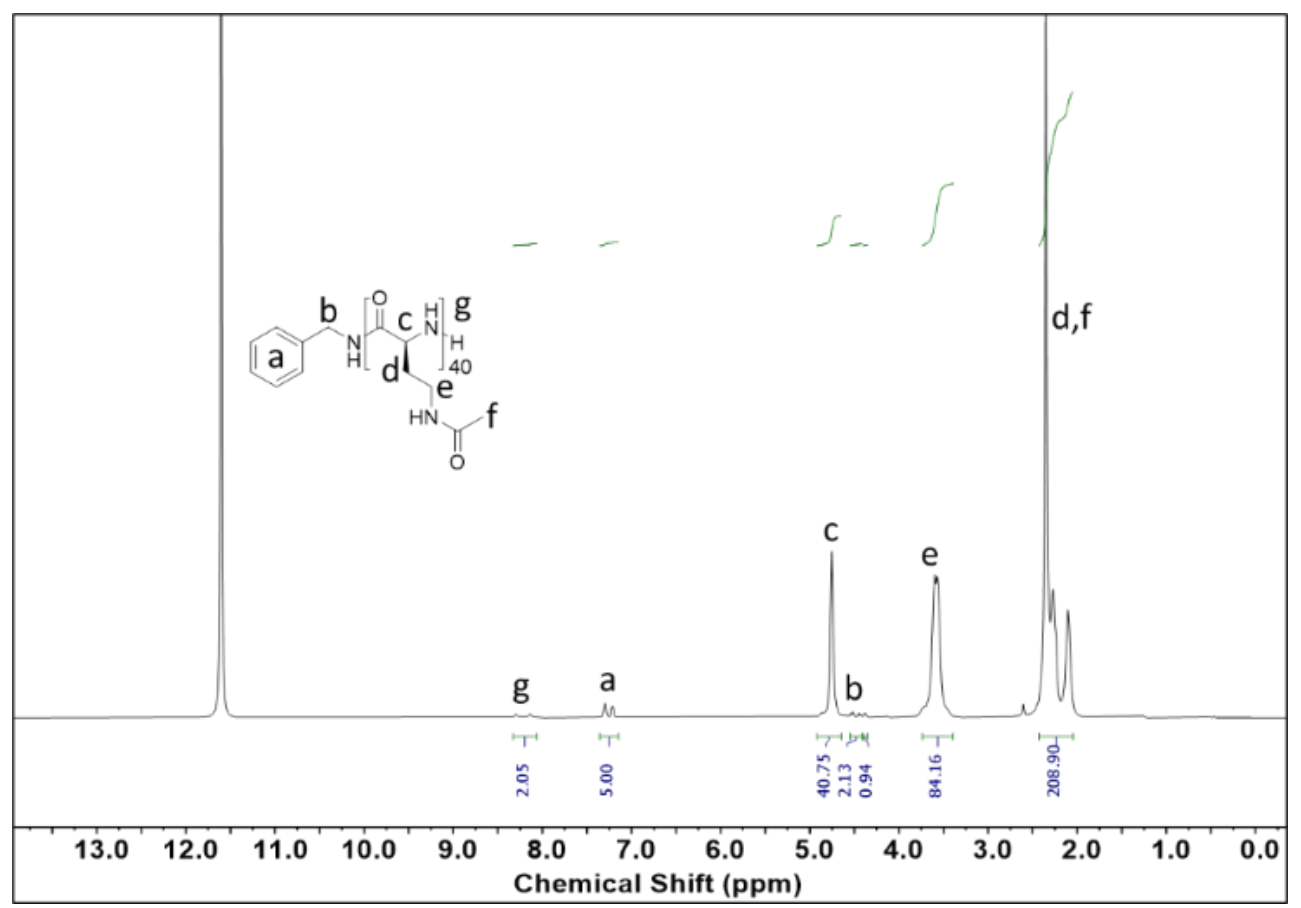

Figure S 4. ${ }^{1 \mathrm{H}} \mathrm{NMR}$ spectra of $\mathrm{P}\left(\mathrm{NADA}_{40}\right)$.

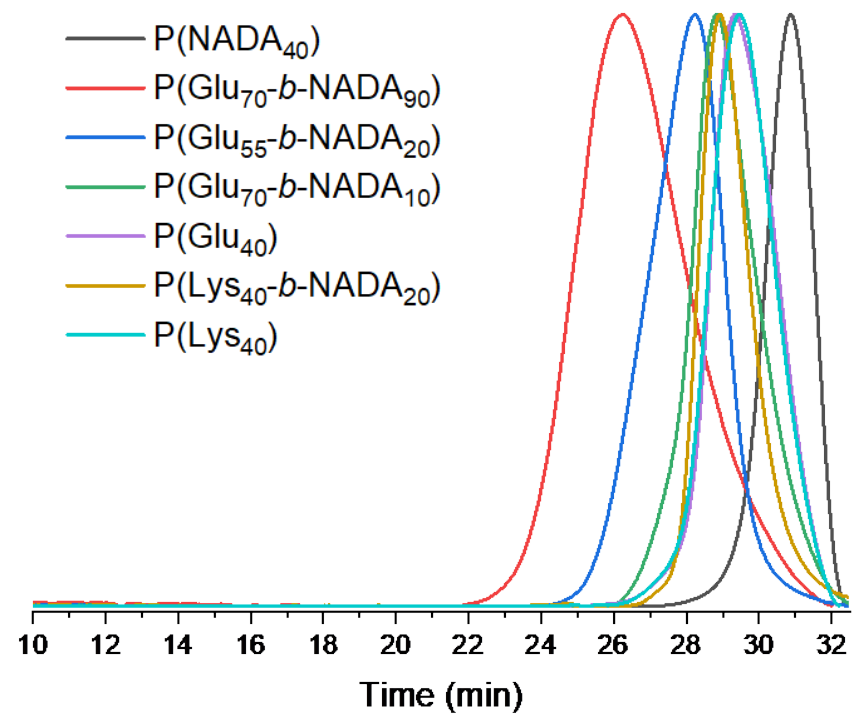

Figure S 5. GPC plots of the synthesized polymers. 


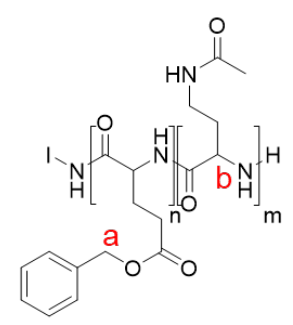

$$
\mathrm{D}_{\mathrm{a}}=1.79 \times 10^{-10} \mathrm{~m}^{2} / \mathrm{s} \quad \mathrm{D}_{\mathrm{b}}=1.76 \times 10^{-10} \mathrm{~m}^{2} / \mathrm{s}
$$

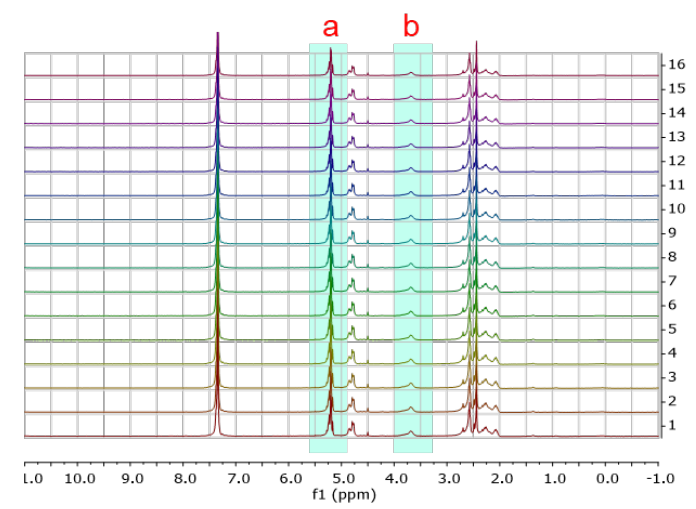

Figure S 6. DOSY NMR of $\mathrm{P}(\mathrm{Glu}-b-\mathrm{NADA})$.

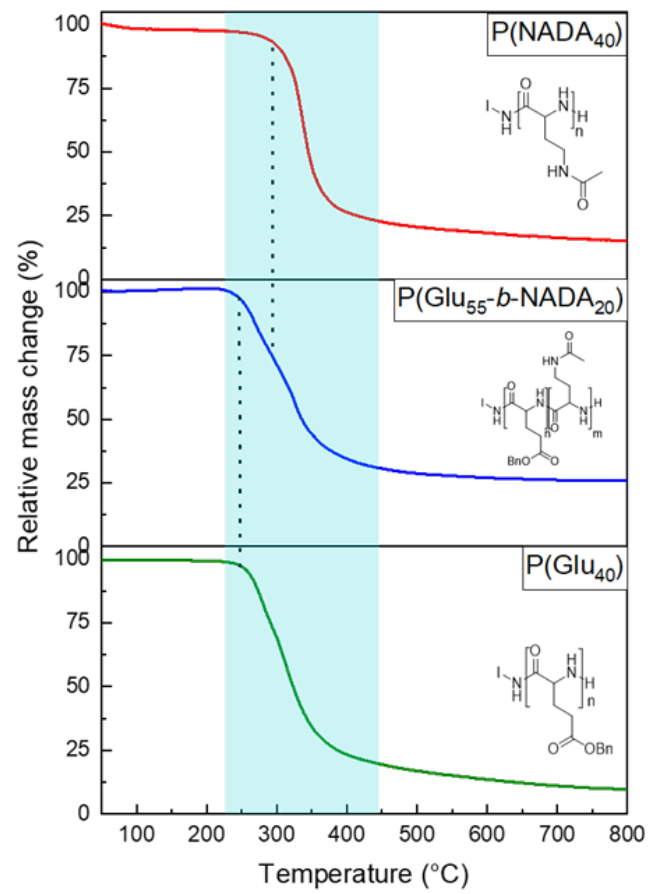

Figure S 7. TGA diagrams of $\mathrm{P}\left(\mathrm{NADA}_{40}\right), \mathrm{P}\left(\mathrm{Glu}_{55}-b-\mathrm{NADA}_{20}\right)$, and $\mathrm{P}\left(\mathrm{Glu} \mathrm{u}_{40}\right)$. 


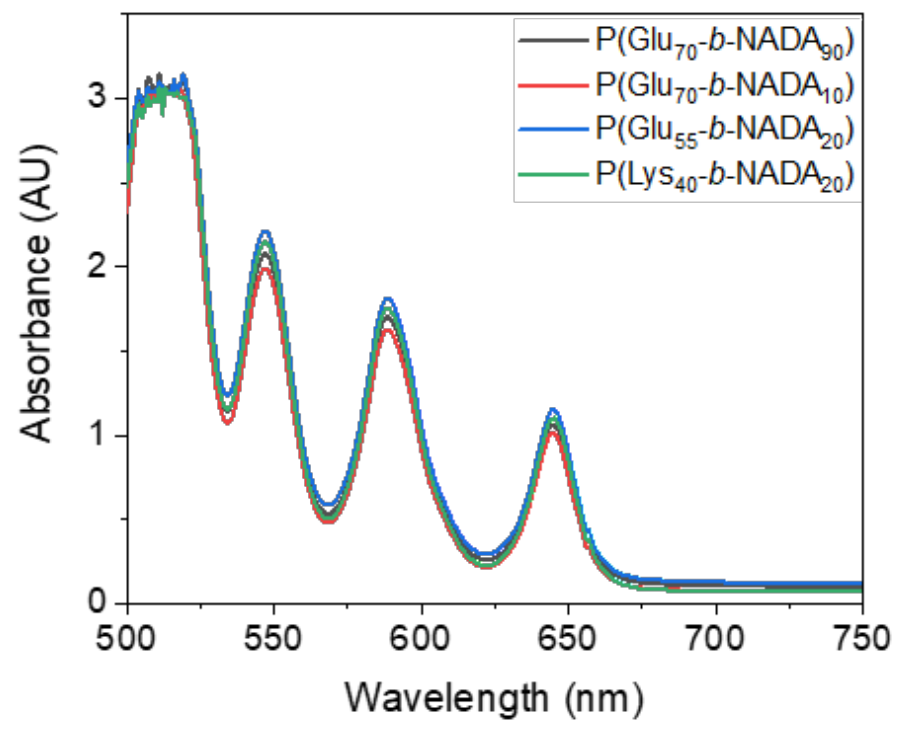

Figure S 8. UV-Vis data of mTHPP-loaded polymers.
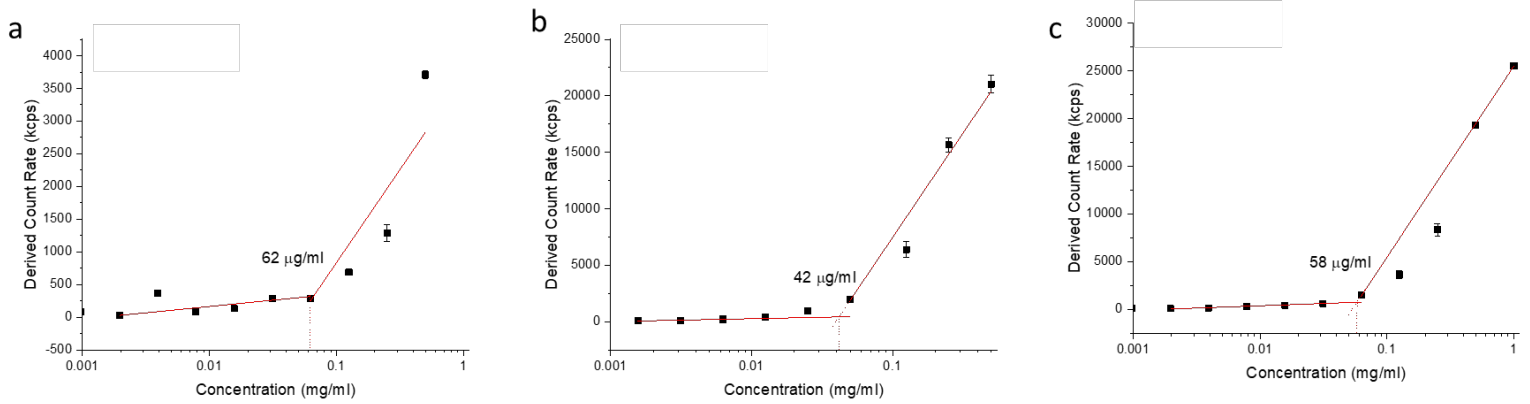

Figure S 9. DLS count rate plotted against concentration for (a) $\mathrm{P}\left(\mathrm{Glu}_{70}-b-\mathrm{NADA} \mathrm{A}_{90}\right)$, (b) $\mathrm{P}\left(\mathrm{Glu}_{70}-b-\mathrm{NADA} \mathrm{A}_{10}\right)$, and (c) $\mathrm{P}\left(\mathrm{LyS}_{40}-b\right.$ $\left.\mathrm{NADA}_{20}\right)$. 

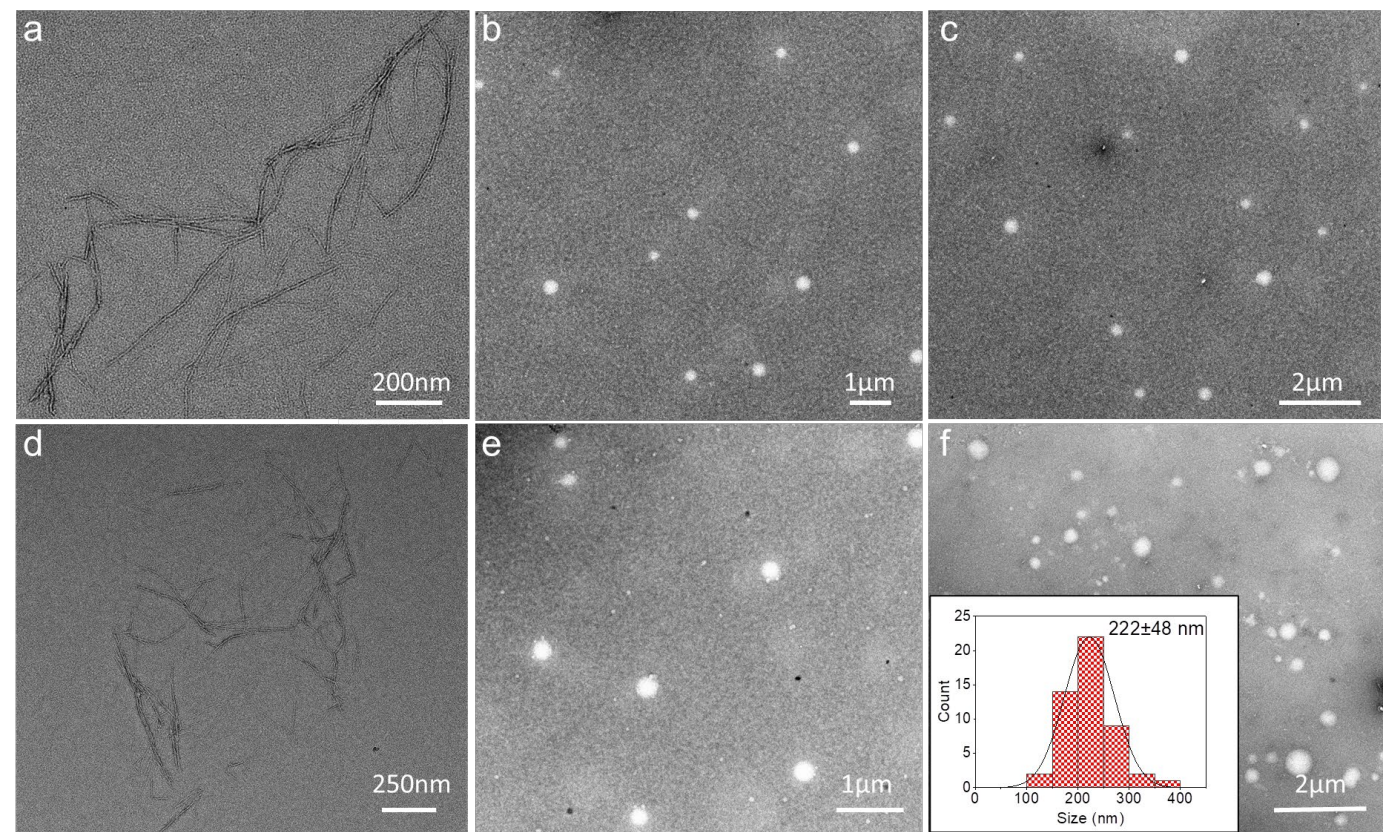

Figure S 10. Transmission electron microscopy (TEM) images of $\mathrm{P}\left(\mathrm{Glu}_{55}-\mathrm{b}-\mathrm{NADA} \mathrm{A}_{20}\right)$ before (a and d) and after self-assembly (b, $\mathrm{c}, \mathrm{e}$, and $\mathrm{f})$.Inset figure $\mathrm{S} 10 \mathrm{f}$ is the size distribution of $\mathrm{P}\left(\mathrm{Glu}_{55}-b-\mathrm{NADA}_{20}\right)$.

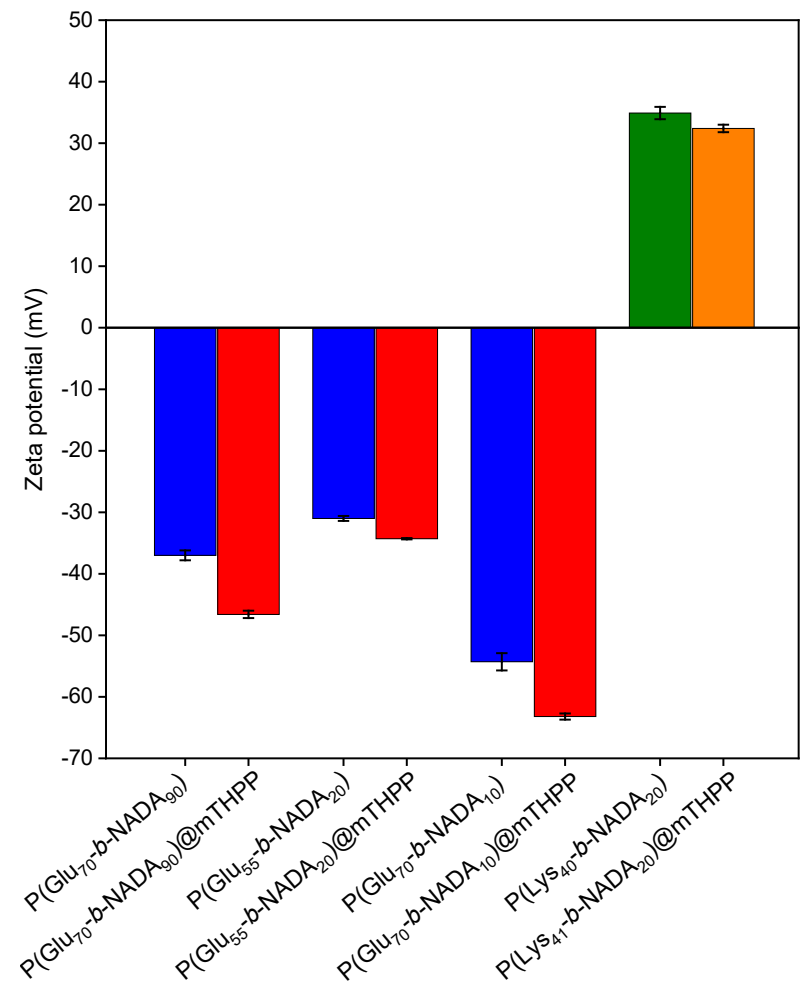

Figure S 11. Zeta potential of synthesized block copolymer before and after loading with mTHPP. 


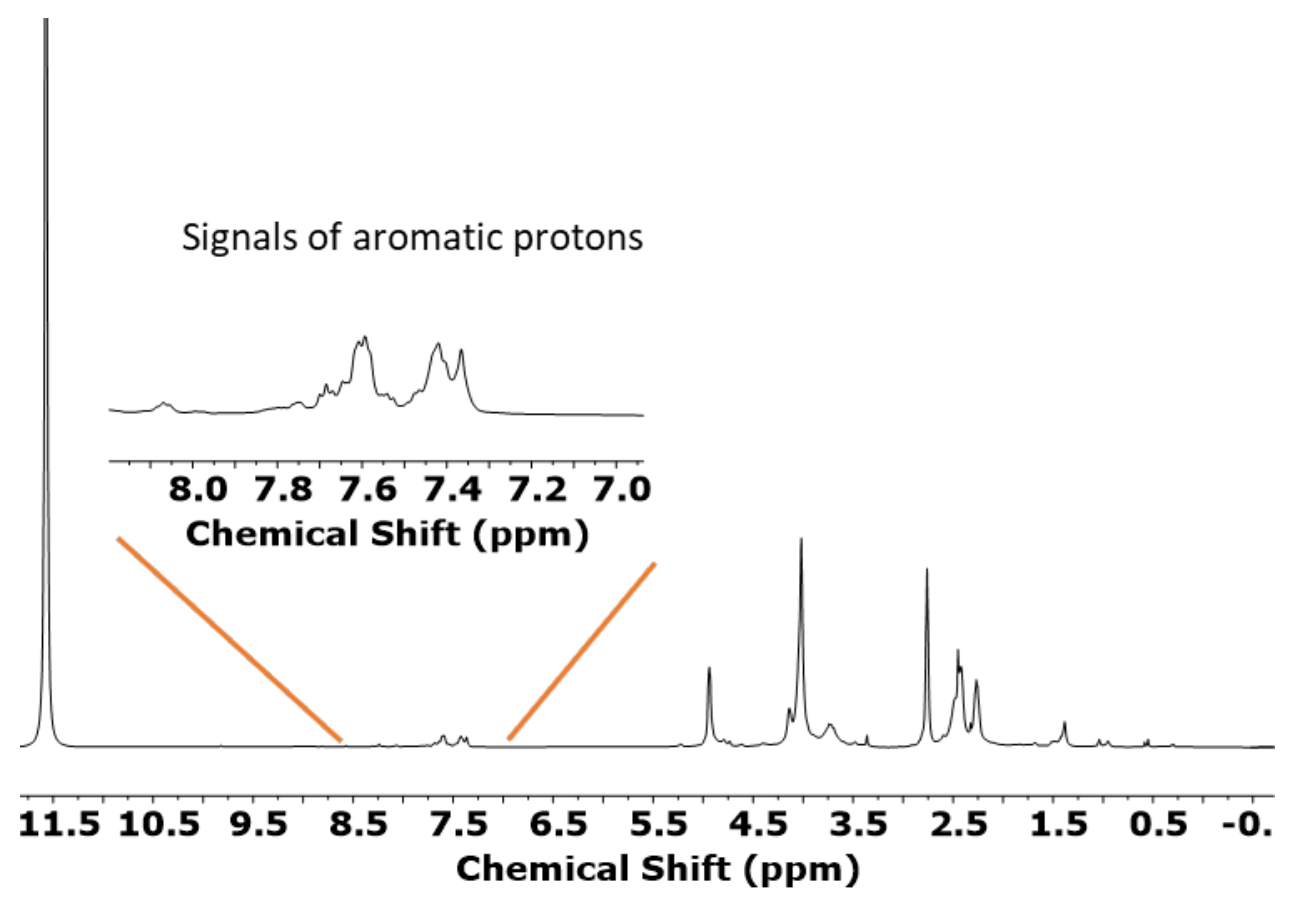

Figure S 12. ${ }^{1} \mathrm{H}$ NMR of $\mathrm{P}\left(\mathrm{Glu}_{55}-b-\mathrm{NADA}_{20}\right)$ labeled with FITC.

a)

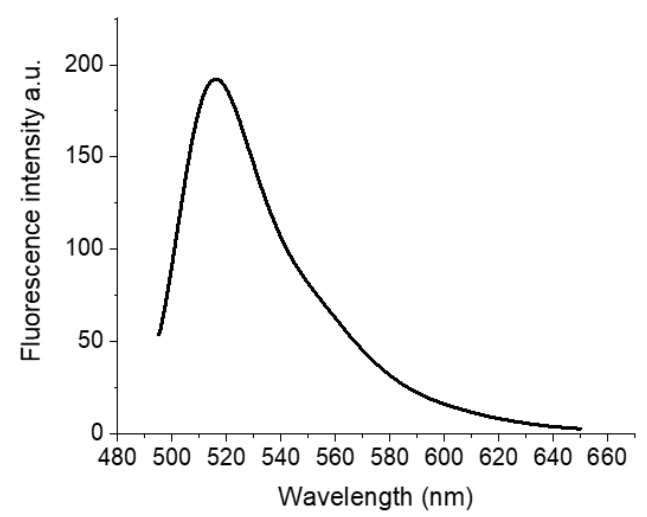

b)

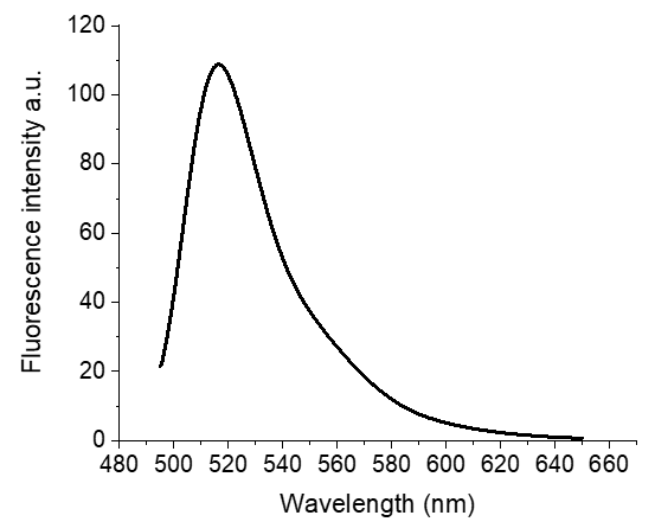

Figure S 13. Fluorescence spectra of (a) $\mathrm{P}\left(\mathrm{Glu}_{55}-b-\mathrm{NADA}_{20}\right)$ and (b) $\mathrm{P}\left(\mathrm{Lys}_{40}-b-\mathrm{NADA}_{20}\right)$ labeled with FITC. 


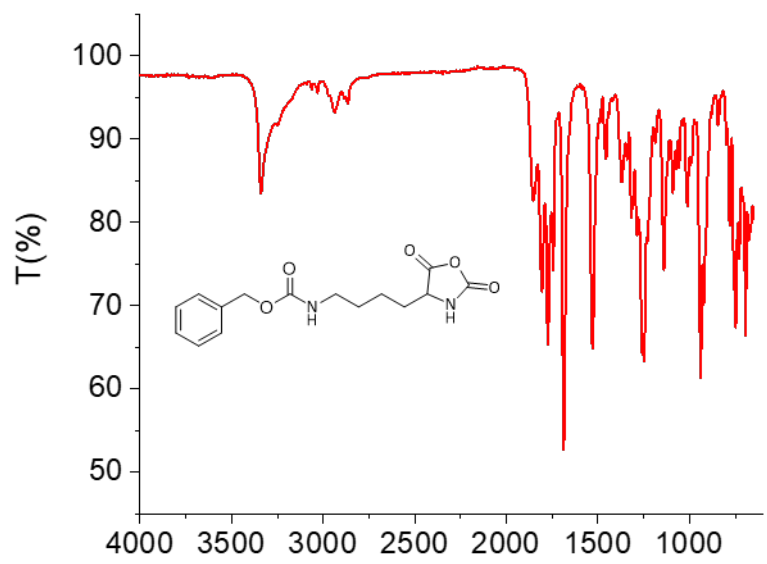

Figure S 14. IR spectra of L-Lys(Z)-NCA,

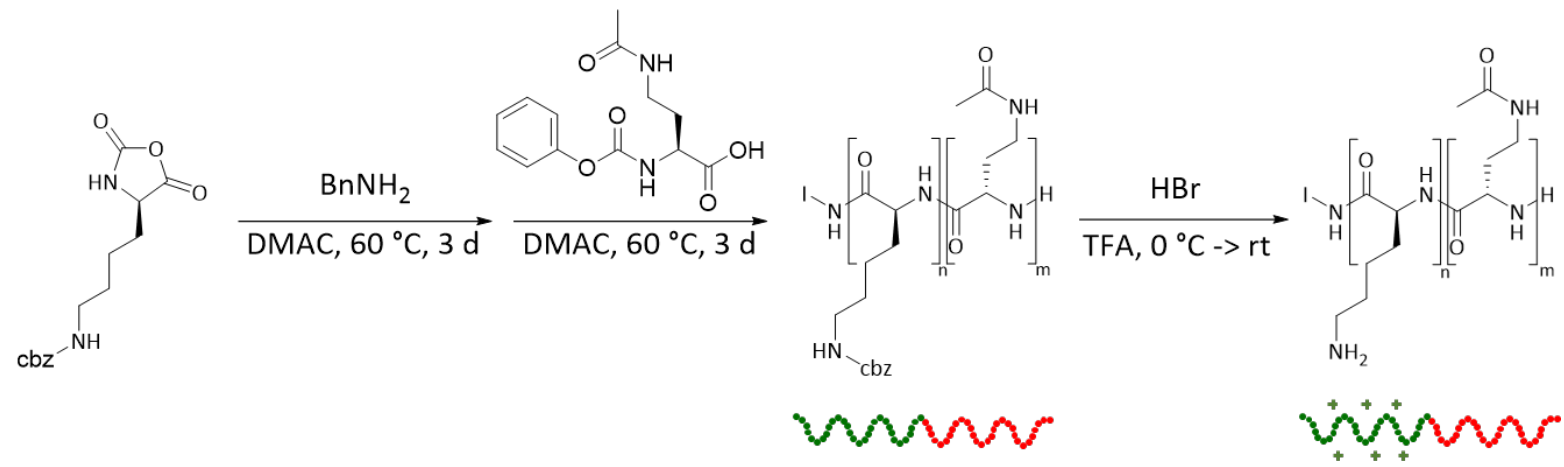

Scheme S2. Synthesis of amphiphilic block co-polypeptides through the combination of ring-opening polymerization of (Lys(Z)-NCA from butylamine initiator and polycondensation of urethane derivative of NADA, Ph-NADA.

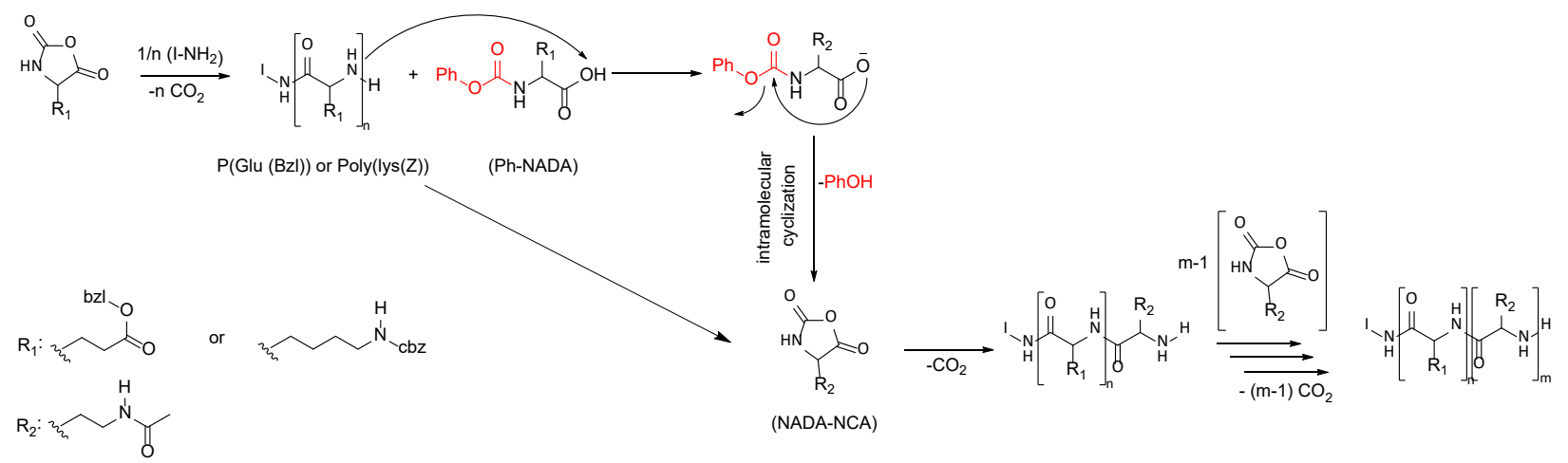

Scheme S3. Mechanism of synthesis of amphiphilic block-co-polypeptides through the combination of ring-opening polymerization of (Glu(Bzl)-NCA or Lys(Z)-NCA from initiator and polycondensation of urethane derivative of NADA, Ph-NADA. 

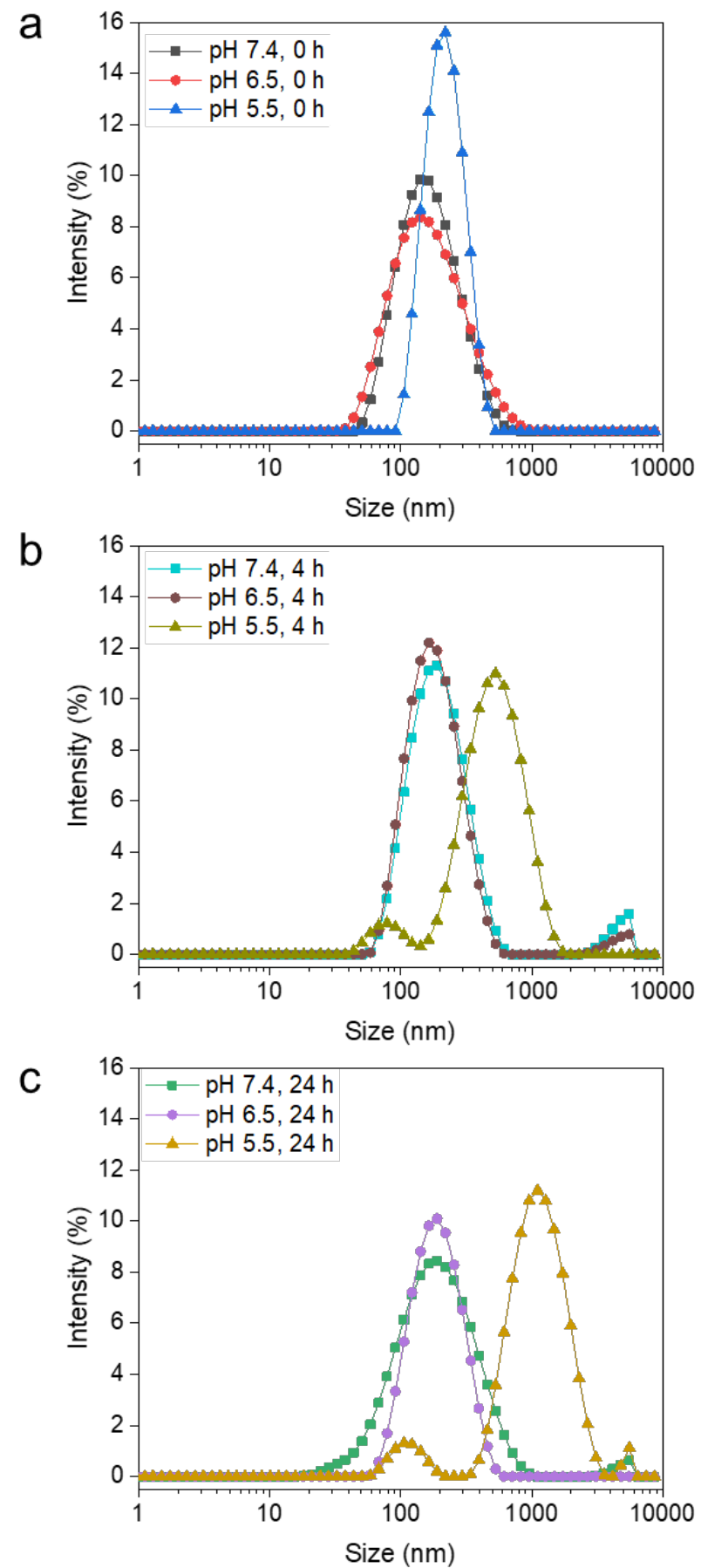

Figure S 15. DLS measurements of $\mathrm{pH}$-induced size changes of $\mathrm{P}\left(\mathrm{Glu}_{55}-b-\mathrm{NADA}_{20}\right)$ after a) 0 hour, b) 4 hour, and c) 24 hours incubation. 